\title{
GUÍA DE AUTORES , REQUISITOS PARA PREPARAR MANUSCRITOS Y PROCESO DE EVALUACIÓN POR PARES.
}

\begin{abstract}
La revista O-Activa de la carrera de Odontología fue creada en el año 2015 y pertenece a la Unidad Académica de Salud y Bienestar de la Universidad Católica de Cuenca. Es un órgano de difusión científica que pretende compartir todo tipo de trabajos con el objetivo fundamental de transmitir el conocimiento dentro del área odontológica, en el cual se incluyen temas de actualidad y relevancia como: Epidemiología, Operatoria Dental, Materiales dentales, Radiología Maxilofacial, Cirugía Bucal y Maxilofacial, Ortodoncia y Ortopedia Maxilar, Odontopediatría, Periodoncia e Implantología, Endodoncia, Rehabilitación Oral, Patología Bucal, Odontología Forense, Salud Pública y aspectos médicos relacionados con la Odontología. La revista va dirigida a todos los estudiantes y profesionales no solo de la especialidad, sino también del área de las Ciencias de la Salud en general. De esta forma se contribuye al fortalecimiento, discusión e intercambio de conocimientos, proporcionando con ello, información que sirva de base para el desarrollo profesional y la formación continua.La presente guía consta de cuatro partes: Tipología de artículos a publicar, requisitos para preparar un manuscrito, instrucciones para la preparación de las secciones del manuscrito y proceso de evluación por pares.
\end{abstract}

\section{TIPOLOGÍA de ARTÍCULOS A PUBLICAR}

\subsection{ARTÍCULO ORIGINAL}

Producto final de una investigación científica cuantitativa o cualitativa, que parte de una pregunta de investigación, toma datos y llega a conclusiones. Las revisiones sistemáticas son consideradas en este rubro. El cuerpo de este artículo se presenta con las siguientes partes: Introducción, MaterialesMétodos, Resultados y Discusión.La extensión máxima del manuscrito debe ser de 12 páginas incluyendo tablas y gráfi$\cos$.

\subsection{REPORTE DE CASO CLÍNICO}

Presentación de un caso o una serie de casos clínicos, que por su poca frecuencia o su tratamiento multidisciplinario vale la pena compartir con la comunidad odontológica a fin de aumentar el bagaje académico-clínico de los lectores. El cuerpo de este artículo se presenta con las siguientes partes: Introducción, Presentación del caso y Discusión.La extensión máxima del manuscrito debe ser de 8 páginas incluyendo tablas y gráficos.

\subsection{CARTAS AL EDITOR}

Comunicación breve (1 página) que sirve para fijar posición frente a un artículo publicado en esta revista y que el lector discrepa parcial o totalmente con lo publicado, se sustenta en evidencia científica. La revista se guarda el derecho de la publicación de las cartas al editor.

\subsection{ARTÍCULOS DE REVISIÓN}

Se consideran en este tipo las revisiones narrativas, que son una exhaustiva búsqueda de información respecto a una pregunta en ciencias de la salud que es difícil de investigar con fuente de datos primaria. No requiere tratamiento estadístico. Por su contenido se convierte en valioso material de consulta. El cuerpo de este artículo se presenta con las siguientes partes: Introducción, Estado del Arte, Discusión y Conclusiones.La extensión máxima del manuscrito debe ser de 12 páginas incluyendo tablas y gráficos.

\subsection{CONTRIBUCIÓN DIDÁCTICA DOCENTE / ARTÍCU- LO DE OPINIÓN}

Artículo que recopila la opinión de un experto en determinado tema, se escribe por invitación del comité editorial de la revista a fin de fijar posición respecto a un tema. Por su contenido se convierte en valioso material de consulta. El cuerpo de este artículo se presenta con las siguientes partes: Introducción, Estado del Arte, Discusión y Conclusiones.La extensión máxima del manuscrito debe ser de 6 páginas incluyendo tablas y gráficos.

\section{REQUISITOS PARA PREPARAR MANUSCRITOS}

Los manuscritos a ser recibidos deben ser estructurados de acuerdo a los Requerimientos Uniformes para el énvio de manuscritos a Revistas Biomédicas elaborabos por el Comité Internacional de Editores de Revistas Médicas cuya versión se encuentra disponible en www.icmje.org. Los autores deben seguir la guía de autores y requisitos específicos de la Revista O- ACTIVA, con respecto a la estructura y tipos de artículos que pueden ser recibidos: 


\subsection{Normas de Estructuración:}

La estructura del manuscrito debe constar de : título en idiomas español e inglés, autores y su afiliación, resumen en idioma español e inglés, palabras clave y key words(máximo 6) que consten en el buscador de descriptores de ciencias de la salud (DECS) disponibles en el enlace http://decs.bvs.br/, cuerpo del artículo con sus respectivas secciones dependiendo del tipo del mismo y referencias bibliográficas con normas Vancouver.

\subsection{Normas de Presentación:}

Todo artículo se recibe en formato .doc o .docx del programa Microsoft Word. El tamaño de hoja es A4, con márgenes $2.5 \mathrm{~cm}$ superior; $3.00 \mathrm{~cm}$ izquierdo; $2.5 \mathrm{~cm}$ derecho y $2.5 \mathrm{~cm}$ inferior. El texto debe ser escrito en tipo de letra Arial tamaño 9. Los títulos y subtítulos deben ir en negrita y en tamaño 11. El árticulo puede ser enviado vía email a la dirección electrónica revistaodontologia@ucacue.edu.ec o entregado personalmente al editor ejecutivo en el departamento de investigación de la carrera de Odontología de la Unidad Académica de Salud y Bienestar mediante un disco compacto, en ambos casos se deben especificar explicitamente los siguientes archivos por separado:

- Carta de Presentación: Dirigida al Director de la Revista, incluirá títulos del artículo , autores y su afiliación institucional de acuerdo a las recomendciones ICMJE, dirección electrónica de los autores, y grados académicos de cada uno de ellos. Uno de los autores debe ser designado autor de correspondencia debiendo aparecer al inicio del artículo su correo electrónico.En este apartado el autor deberá especificar adicionalmente los siguientes requisitos por separado:

* Detallar los criterios que se tuvieron para la autoría y responsabilidad de los autores por el contenido del artículo (se consideran autores aquellos que han participado en la concepción del estudio, redacción del artículo o revisón del mismo y levantamiento de correcciones hasta la aprobación final del documento). El orden de los autores es responsabilidad de autor principal.

* Grado de contribución de cada autor o declaración de los autores según su contribución para definir el orden de mención en caso que el autor principal no lo haya predefinido. Esta información debe presentarse adicionalmente en el artículo al final, despúes de la bibliografía.

* Quienes por su grado de contribución no puedan ser considerados como autores, pueden ser nombrados en agradecimientos dado que participaron en la investigación con ayuda técnica, logística y operativa.

* Fuentes de financiamiento: que permitieron la realización del estudio, trabajo o manuscrito. Si no existe financiamiento se deberá especificar que es asumido por los autores (autofinanciado). Este parámetro deberá adicionalmente ser mencionado en el apartado correspondiente del artículo( ej. materiales y métodos).

* Conflictos de interés: Cada uno de los autores deberá efectuar una declaración de posibles conflictos de interés. Todos los conflictos de interés deben ser totalmente descritos en el apartado correspondiente del artículo. Existe un conflicto de interés cuando un autor o la institución a la que pertenece el mismo tiene relaciones o compromisos duales personales o financieros que podrian influir de forma poco adecuada (sesgo) en sus acciones.

* Aspectos éticos de la investigación cuando se trata de experimentos con seres humanos es necesario presentar la aprobación de un comité de ética de investigación en seres humanos (CEISH) reconocido por el Ministerio de Salud Pública del Ecuador o del país de origen del estudio. En el contenido del artículo deberá constar explícitamente el formulario del consentimiento informado así como la autorización de la entidad donde se realizó el estudio.

* Declaración de originalidad y carácter inédito del artículo, no se aceptarán manuscritos que se han presentado simultaneamente en otras revistas científicas (envio duplicado o redundante), a menos que se especifíque la republicación por decisión del autor y del editor.

* En el caso de estudios experimentales con seres humanos, es necesario presentar el registro de estudios de ensayos clínicos ejecutados en el país con su aprobación obligatoria y registro de la Agencia Nacional de Regulación, Control y Vigilancia Sanitaria (ARCSA). Los ensayos clínicos no ejecutados en Ecuador, deberán indicar el número de registro internacional del estudio y el comité de ética responsable de la aprobación.

El autor podrá encontar un modelo de esta carta en la página web de la revista (http://oactiva.ucacue.edu.ec/).

- Texto del artículo : título del artículo en idiomas español e inglés, autores y su afiliación institucional (ICMJE), resumen en idiomas español e inglés, palabras clave en idioma español e inglés (DECS), desarrollo o cuerpo del artículo en secciones, referencias bibliográficas en estilo Vancouver y grado de contribución o declaración de los autores según su contribución.

- Gráficos, ilustraciones y diagramas: de ser el caso enviar los archivos originales en formato CMYK en resolución mínima de 300ppp. Adicionalmente en el cuerpo del artículo debe figurar el gráfico en un formato de menor calidad, con la finalidad de sugerir esta ubicación al editor de la revista. La cantidad permitida por documento es de máximo 6 tablas, 6 gráficos y 2 diagramas. Al pie de cada uno de estos se debe especificar la autoría o fuente. 


\subsection{Normas para citas Bibliográficas:}

Todo el texto, tablas y gráficos se acatarán y redactarán conforme a los requisitos de uniformidad para manuscritos presentados en revistas biomédicas, adaptados por la National Library of Medicine, de acuerdo a las recomendaciones del Comité Internacional de Directores de Revistas Biomédicas (ICMJE), para ello se detalla la Guía de Referencia para Citas Bibliográficas.

Las referencias deberán ser numeradas consecutivamente en el órden en el cual son mencionadas en el texto. Identifique referencias en el texto, tablas y leyendas por números árabes con superíndices. Las páginas deben ser numeradas en el extremo inferior derecho.

\subsubsection{Guía de Referencia para Citas Bibliográficas.}

La Revista Odontología Activa exige las normas de Vancouver para la elaboración de la bibliografía usando referencia del texto únicamente con número en superíndice separados con comas y cuando presentan varias seguidas se coloca el primer y el último número separados por guión (ejemplo, 1, 4-8) siguiendo el orden utilizado de manera ascendente. La referencia en cuadros o figuras se numeran según el orden presentado en el texto previamente. La revista ha establecido como número mínimo 12 referencias de revistas indexadas de las cuales 4 o más deben ser de artículos de Latinoamérica.

Se emplea el estilo de referencias y de abreviatura de las revistas recomendado por la U.S. National Library of Medicine (NLM) usa en el Index Medicus. Revisar lista y recomendación en: http://www.nlm.nih.gov

Las referencias llevan: el primer apellido de cada autor con su primera letra en mayúscula, seguido por la inicial del primer nombre, título del artículo, nombre abreviado de la revista (revisar en bases de referencias), mes, año de publicación, volumen, número en paréntesis y el intervalo de páginas separadas por un guión intermedio.

Se recomienda a los autores confirmar referencias con los artículos originales, evitar usar resúmenes y aclarar cuando se utilizan artículos aceptados en proceso de publicación.

En caso de tener autores con guiones entre dos apellidos recomendamos mantener un criterio fijo de usar un guion entre dos apellidos o solo un apellido en todas las referencias para no llevar a confusiones. Cuando existan las preposiciones "Von", "Van", "Di”, "De", "De la " se citará antes del apellido.

La disposición de los autores se transcribirá en el orden que se encuentren en los artículos. Escribiendo su primer apellido y la inicial de su primer nombre hasta un número de seis autores; en caso de presentar un número mayor se escribirá seis autores y posteriormente se colocará et al (en cursiva).

\section{Artículo de Revista}

Solar P, Geyerhofer U, Traxler H, Windisch A, Ulm C, Watzek G. Blood supply to the maxillary sinus relevant to sinus floor elevation procedures. Clin Oral Implants Res. 1999; 10 (1): 34-44.

\section{Artículo con más de seis autores}

Gröbe A, Eichhorn W, Hanken H, Precht C, Schmelzle $\mathrm{R}$, Heiland $\mathrm{M}$ et al. The use of buccal fat pad (BFP) as a pedicled graft in cleft palate surgery. Int J Oral Maxillofac Surg. 2011; 40: 685-689.

\section{Folleto con Autor corporativo}

Secretaría de Salud de México. Situación actual de la tuberculosis en el Mundo, México, Veracruz. Avances y Desafíos. Secretaría de Salud de México 2011.

\section{Artículo Sin autor}

Cancer in South Africa (editorial). S. Afr Med J 194;84:15.

\section{Artículo en otro idioma distinto del inglés}

Si el Artículo es derivado del latín se coloca en su idioma original, si no lo es debe traducirse al Ingles usando un traductor oficial en la página de Medline. El resultado de la traducción colocar en ingles en corchetes seguido del idioma o dialecto original.

Autor A, Autor B. [Artículo en Alemán traducido al Español]. Artículo en Alemán. año; volumen (\#): pag-pag.

\section{Volumen con suplemento}

Autor A, Autor B. Título. Revista. Año; Vol, Suppl \#: pagpag.

\section{Número con suplemento}

Autor A, Autor B. Título. Revista. Año, Vol. (1 Suppl 2): pag-pag.

\section{Volumen con parte}

Autor A, Autor B. Título. Revista. Año; vol (Pt \#): pag-pag.

\section{Número con parte}

Autor A, Autor B. Título. Revista. Año; vol (Número Pt \#): pag-pag.

\section{Número sin volumen}

Autor A, Autor B. Título. Revista. Año; (número 35-36): pagpag. 


\section{Sin número ni volumen}

Autor A, Autor B. Título. Revista. Año: pag-pag.

\section{Páginas en números romanos}

Autor A, Autor B. Título. Revista. Año; Vol(): xi-xiii

\section{Mapa}

North Carolina. Tuberculosis rated per 100,000 population, 1990 [demographic map]. Raleigh: North Carolina Dept. of Environment, Health, and Natural Resources, Div. of Epidemiology; 1991.

\section{Diccionario y obras de consulta semejantes}

Autor A, Autor B. Nombre del diccionario. Ciudad, País: Editorial, Año.

\section{Material electrónico \\ Artículo de revista en formato electrónico Autor A, Autor B. Título. Revista [serial online]. Año Abrevia- tura Mes-Abreviatura Mes; [cited año Abreviatura del Mes ]; Vol (\#): [\# screens]. Available from: URL: http://www.coo.gov/ndod/ED/eid.htlm}

\section{Libros}

\section{Individuos como autores}

Miloro M, Kolokythas A. Management of complications in oral and maxillofacial surgery $1^{\mathrm{a}}$ ed. Wiley-Blackwell, 2012. Indicación: La primera edición puede o no colocarse. Si existe más de un volumen debe colocarse la abreviatura de Vol. \#

\section{Capítulo de libro}

Patel P, Bagheri S. Penicillin allergy/ Anaphylaxis In: Bagheri S, Jo C. Clinical review or oral and maxilofacial surgery. Missuri: Elsevier, 2008: 22-25.

\section{Tesis}

Riera L, Ramos R. Relación de la autopercepción de necesidad de tratamiento ortodóntico con prevalencia de maloclusiones en escolares de 12 años en San Blas- Cuenca 2016 (tesis de licenciatura en odontología). Ciudad de Cuenca. Universidad Católica de Cuenca. 2016.

\section{INSTRUCCIONES PARA LA PREPARACIÓN DE LAS SECCIONES DEL MANUSCRITO}

Los requisitos para la presentación de manuscritos dentro de las secciones de todos los diseños de los estudios y formatos son los siguientes:

\subsection{PORTADA O PÁGINA DEL TÍTULO}

La cual incluirá información general acerca del artículo:

- Título en español e inglés.

- Información sobre autores: nombres de autores, grados académicos más altos de cada persona, afiliaciones institucionales u organizaciones a las que el trabajo debe ser atribuido. Información de contacto completa, incluyendo el correo postal y el electrónico, figurando en la portada el teléfono de los autores y números de fax. Adicionalmente se debe especificar el autor de correspondencia.

- Especificar los agradecimientos por el apoyo brindado para la realización del estudio.

- Limitaciones de responsabilidad y declaración de un autor sobre su responsabilidad en los puntos de vista, liberando de responsabilidad a la institución en la que trabaja o a la fuente de financiamiento.

- Fuentes de apoyo, subvenciones, equipos, medicamentos o cualquier otro apoyo que facilitó la realización de la investigación.

- Recuento de palabras que contiene el texto del artículo excluyendo resumen, agradecimientos, títulos de tablas y figuras, y bibliografía, con la finalidad de garantizar que la extensión del manuscrito está dentro de los formatos de la revista.

- Especificar el número de figuras y tablas, para confirmar que todas las figuras y tablas serán incluidas en el manuscrito.

- Declaración de conflicto de interés por parte de cada autor.

\subsection{HOJA DE RESUMEN Y ABSTRACT}

El resumen debe proporcionar el objetivo del estudio, los materiales y métodos, resultados principales y conclusiones principales. El resumen debe contener un máximo de 250 palabras en un solo párrafo. Se ajustan a esta estructura de resumen, los artículos originales, las revisiones de literatura. Los otros tipos de artículos deben presentar un resumen que en lo posible se ajuste a esta norma.

En la misma página se debe presentar el Abstract, que es la traducción del resumen al idioma inglés. De igual forma debe ser redactado en 250 palabras como máximo.

\subsubsection{PALABRAS CLAVE Y KEY WORDS}

Pueden ser simples ("ortodoncia") o compuestas ("calidad de vida") y deben estar presentadas a continuación del contenido del resumen o abstract; son de ayuda para que los lectores encuentren un artículo en particular. Las palabras clave en idioma inglés y español deben verificarse en base a los descriptores de ciencias de la salud (DECS).

\subsection{TEXTO DEL ARTÍCULO}

\subsubsection{INTRODUCCIÓN}

Incluye el contexto del estudio, el propósito de la investigación, los antecedentes o estudios anteriores, la definición 
de la variable, se menciona brevemente el instrumento de investigación y se finaliza con el objetivo. Todos estos parámetros con su respaldo bibliográfico. Se deben mencionar o citar sólo referencias necesarias. Pueden mencionarse brevemente la conclusión general del trabajo.

\subsubsection{MATERIALES Y MÉTODOS}

En el caso de estudios observacionales esta sección debe contar con: el diseño del estudio, el lugar y tiempo en el que se tomaron los datos, los criterios de inclusión de unidades de estudio, la forma que se calculó el tamaño de muestra, la forma de medición de las variables, los potenciales sesgos, los métodos estadísticos que se emplearon y los aspectos éticos, indicando que la investigación respetó los principios de la Declaración de Helsinki.

En el caso de estudios experimentales esta sección debe contar con: diseño del ensayo, criterio de selección de participantes, procedencia y si hubo aleatorización, detalle suficiente de las intervenciones, especificación de la medición de las variables, la estrategia de determinación del tamaño de muestra, si se utilizó el enmascaramiento o cegamiento, métodos estadísticos utilizados, posibles sesgos y los aspectos éticos, indicando que la investigación respetó los principios de la Declaración de Helsinki.

\subsubsection{RESULTADOS}

Se recomienda organizarlos desde lo más simple hasta lo más complejo, la manera de comprenderlos mejor es usando tablas y figuras. No repita todos los datos de las tablas o figuras en el texto, destaque o resuma sólo las observaciones más relevantes. Los materiales extra o suplementarios y detalles técnicos pueden ser publicados en un anexo accesible, es decir sin interrumpir el flujo del texto, o ser publicados únicamente en la versión electrónica.

\subsubsection{DISCUSIÓN}

En esta sección se analizarán los resultados del estudio tomando como referencia la pregunta de investigación o el objetivo general; también incluirá la síntesis del problema estudiado, los procedimientos realizados para resolverlo, lo que se esperaba encontrar y por qué; se enfocará en aspectos importantes del estudio con sus conclusiones.

En el caso de los artículos originales y los reportes de casos clínicos las conclusiones deben estar explicitadas en esta sección.

\subsubsection{CONCLUSIONES}

Incluye deducciones específicas del estudio y hace referencia a los descubrimientos, sin que estos sobrepasen la evidencia. Se presenta como una sección propia en los artículos de revisión de la literatura, artículos de opinión y contribución didáctica docente.

\subsubsection{RECOMENDACIONES}

Las recomendaciones pueden orientarse en el campo correspondiente a su investigación; de esta forma existe la posibilidad de nuevos estudios con sus respectivos diseños de investigación o de la aplicación de los datos del artículo en el tratamiento de pacientes. No es una sección obligatoria.

\subsection{ANEXOS}

\section{Pueden ser:}

- Tablas, en éstas se pueden observar resultados descriptivos numéricos.

- Gráficos estadísticos, muchas veces son más explicativos que las tablas.

- Figuras, se refieren a imágenes originales del estudio.

Para enumerar las tablas y figuras se realizará de forma independiente, con un máximo de 14 anexos.

Cada anexo debe tener en su encabezado el tipo y número de anexo, continuando con el título y posteriormente se insertará la figura pertinente; al final del anexo se puede incluir abreviaturas o códigos para una mejor comprensión de los lectores.

\section{PROCESO DE EVALUACIÓN POR PARES}

Para enviar un manuscrito a la revista O-Activa se debe remitir a la siguiente dirección de correo electrónico institucional: revistaodontologia@ucacue.edu.ec. Es posible también recibirlos de manera directa en el Departamento de Investigación de la Carrera de Odontología de la Universidad Católica de Cuenca, a través del contacto personal con el Cuerpo Editorial. La revisión de los artículos consta de dos procesos:

- Verificación del cumplimiento del formato de la revista: se comprueba que el artículo cumpla con todos los requisitos de forma, presentación, coherencia, gramática y metodología, este procedimiento no garantiza que el artículo sea aceptado para su publicación. Una vez que el artículo cumpla los requisitos de forma solicitados en la guía de autores y preparación de manuscritos, se procede a dar ejecución al siguiente proceso.

- Revisión por pares o juicio de pares externos se evalúa la temática, originalidad, interés, aporte, validez, beneficios y la calidad de la presentación del manuscrito. Cada artículo es sometido a una revisión anónima por dos pares externos aleatorizados con el sistema de doble ciego,quienes disponen de 72 horas para la evaluación del artículo asignado. Los dictámenes finales de los pares académicos tienen las siguientes posibilidades: "Publicar,sin cambios" "Publicar con cambios menores" "Necesita ser revisado nuevamente" "Rechazado". 
Para culminar, cualquier corrección de un artículo aprobado, será consensuado con el autor principal de la publicación y después el Consejo Editorial Interno decidirá en que volumen y número de la revista será incluida la publicación, notificándo dicho proceso a los autores a través de una carta de aceptación. 